\title{
The QCD Scale in the Heavy Quark Expansion *
}

\author{
Lisa Randall ${ }^{\dagger}$ and Eric Sather \\ Massachusetts Institute of Technology \\ Cambridge, MA 02139
}

\begin{abstract}
We argue that consistency of the combined heavy quark and chiral effective lagrangian requires the QCD scale which multiplies $1 / M$ in the heavy quark expansion to be the chiral symmetry breaking scale, $\Lambda_{C S B}$, rather than the QCD scale, $\Lambda_{Q C D}$. This means that either there is large uncertainty in the accuracy with which the heavy quark effective theory can be applied to $c$ quarks or the cutoff scale of the heavy quark chiral effective theory is lower than has been assumed.
\end{abstract}

${ }^{*}$ This work is supported in part by funds provided by the U.S. Department of Energy (DOE) under contract \#DE-AC02-76ER03069 and in part by the Texas National Research Laboratory Commission under grant \#RGFY92C6.

${ }^{\dagger}$ National Science Foundation Young Investigator Award.

Alfred P. Sloan Foundation Research Fellowship.

Department of Energy Outstanding Junior Investigator Award.

CTP\#2167

November 1992 


\section{Introduction}

The heavy quark theory [1] tells us that in the limit that quarks are infinitely massive, symmetries exist between quarks of different flavor and spin. Of course, in the real world, quarks are not infinitely massive, so finite mass corrections must be taken into account. This means that there are corrections to the symmetry relations (aside from calculable QCD corrections) which are suppressed by factors of $1 / M$, where $M$ is the mass of the heavy quark. There must be a dimensionful factor to compensate this mass suppression. Although we don't know precisely the scale, we know that it is some QCD scale, which the heavy quark expansion takes to be of order $\Lambda_{Q C D}$, about a few hundred $\mathrm{MeV}$ [2].

Recent work [3] has incoporated low energy chiral dynamics into a heavy quark theory. In this theory, one can consider the interactions of low energy pions and kaons with the heavy mesons. In this note, we argue that consistency of the chiral and heavy quark expansions requires that the QCD scale which multiplies $1 / M$ in the heavy quark expansion is the chiral symmetry breaking scale, $\Lambda_{C S B}$, rather than $\Lambda_{Q C D}$.

\section{The QCD Scale}

We present two arguments that the scale appearing in the heavy quark expansion should be $\Lambda_{C S B}$ and not $\Lambda_{Q C D}$. The first argument is a straightforward extension of the usual one-loop argument about the chiral symmetry breaking scale. That is, it is an application of naive dimensional analysis (NDA) [4] to the heavy quark chiral theory. The second argument is based on the assumption that in the chiral lagrangian there is a consistent expansion in flavor symmetry breaking; that is, effects depending on the current quark mass, $m$, of a light quark, should be suppressed by $m / \Lambda_{C S B}$ relative to the leading terms. 
Consider as an example the heavy quark operator

$$
\mathcal{O}^{h}=\bar{h}^{i} \frac{D^{2}}{2 M_{i}} h^{i},
$$

where $i$ is the flavor of the heavy quark. This operator will match onto $1 / M$ suppressed terms in the effective theory of the heavy meson, $H_{i}$. One of these terms will be

$$
\mathcal{O}_{1}{ }^{H}=\operatorname{Tr} \bar{H}^{i} \frac{\partial^{2}}{2 M_{i}} H_{i} .
$$

Another will be the tree level term

$$
\mathcal{O}_{2}{ }^{H}=\frac{\Lambda^{2}}{2 M_{i}} \operatorname{Tr} \bar{H}^{i} H_{i} .
$$

By consistency, we can estimate what $\Lambda$ should be.

We assume the by now standard heavy meson effective lagrangian, given by

$$
\begin{aligned}
\mathcal{L} & =\sum_{i}\left\{-i \operatorname{Tr}\left[\bar{H}_{a}^{i} v_{\mu} \partial^{\mu} H_{i}^{a}\right]+\frac{i}{2} \operatorname{Tr}\left[\bar{H}_{a}^{i} H_{i}^{b}\right] v_{\mu}\left(\xi^{\dagger} \partial^{\mu} \xi+\xi \partial^{\mu} \xi^{\dagger}\right)_{b}^{a}\right. \\
& \left.+\frac{i g}{2} \operatorname{Tr}\left[\bar{H}_{a}^{i} H_{i}^{b} \gamma_{\mu} \gamma_{5}\right]\left(\xi^{\dagger} \partial^{\mu} \xi-\xi \partial^{\mu} \xi^{\dagger}\right)_{b}^{a}\right\}
\end{aligned}
$$

where we have summed over heavy quark flavors. Here $H_{a}^{i}$ is the heavy meson field with heavy quark index $i$ and light quark index $a, v$ is its velocity, and $\xi=\exp \left(i \pi^{\alpha} T^{\alpha} / f_{\pi}\right)$.

Now consider mass suppressed corrections to the heavy meson current, given by

$$
L_{j}^{\nu i}=\operatorname{Tr} \bar{H}^{i} \gamma^{\nu}\left(1-\gamma_{5}\right) H_{j}
$$

At tree level, the matrix element of the current will also contain mass suppressed terms, proportional to $\Lambda^{\prime} / M$. We estimate what we expect the loop calculation to contribute to such a mass suppressed operator. We estimate the contribution from the diagram in which there is one insertion of the current, $L_{j}^{\nu i}$ and one insertion of $\mathcal{O}_{1}{ }^{H}$ on the heavy 
meson line and a pion is emitted and absorbed through the axial coupling proportional to $g$. Because we are interested in determining the scale which multiplies $1 / M$, we reason as was done previously 4 , 4 and assume a cutoff regulator. Recall that the reasoning there was to estimate the one-loop contribution to a given operator, and to choose the chiral symmetry breaking scale in such a way that loop renormalization of a counterterm did not exceed the tree value. The reasoning here is slightly different, because we assume we know that the cutoff scale of chiral dynamics is $\Lambda_{C S B} \approx 4 \pi f_{\pi}$, and from this we wish to determine the tree term in the lagrangian. The assumption is however the same; we do not want a loop contribution to exceed the tree level coefficient. A naive estimate of the loop yields a correction to the original current with coefficient of order $\Lambda_{C S B}^{3} /\left(16 \pi^{2} f_{\pi}^{2} M\right)$. Here, the $1 /\left(16 \pi^{2}\right)$ comes from the loop, the factor of $1 / M$ from the insertion of the mass suppressed operator, and the $1 / f_{\pi}^{2}$ from the pion couplings. To get the right dimensions requires three factors of the cutoff in the numerator. In order that the loop amplitude does not exceed the tree amplitude significantly, we must have $\Lambda^{\prime}=\Lambda_{C S B}$, that is the contribution to the matrix elements of mass suppressed operators is determined by the chiral symmetry breaking scale, $\Lambda_{C S B}$.

Similar reasoning would imply that $\Lambda$ in eqn. (3) is also $\Lambda_{C S B}$. This would follow from the estimate of a one-loop diagram with $\mathcal{O}_{1}{ }^{H}$ inserted. The same dimensional factor $\Lambda_{C S B}$ was used by Georgi in a recent paper [6], where he employed NDA.

For those who are unhappy with estimates based on a cutoff regulator, we present an alternative argument based on the assumption that a consistent chiral expansion incorporates $\mathrm{SU}(3)$ symmetric operators, with symmetry breaking operators suppressed by explicit chiral symmetry breaking factors. One of these factors is the quark mass matrix $m_{q}$, which is a diagonal matrix proportional to the light quark masses, $m_{u}, m_{d}$, and $m_{s}$. From the usual chiral lagrangian expansion, we know that these dimensionful symmetry breaking parameters must 
occur suppressed by the chiral symmetry breaking scale, $\Lambda_{C S B}$, relative to the leading term. Using this fact, we once again show that the scale $\Lambda$ must be taken as $\Lambda_{C S B}$ for consistency.

This sort of calculation was considered in ref. [9], where a large radiative correction to the $1 / M$ suppressed chromomagnetic operator was generated at one loop. Although it is not necessary, the argument here is simplest if we insert the operator $\mathcal{O}_{1}{ }^{H}$ not once but twice, to generate a correction to the current at order $1 / M^{2}$. Using dimensional regularization, one would generate a correction to the current proportional to the relevant mass scale internal to the loop raised to the appropriate power. To be specific, let's consider the kaon loop contribution, which will be proportional to $m_{K}^{4} /\left(\Lambda_{C S B}^{2} M_{c}^{2}\right) \approx m_{s}^{2} / M_{c}^{2}$. We rewrite this to make the chiral expansion parameter explicit as $\left(m_{s} / \Lambda_{C S B}\right)^{2}\left(\Lambda_{C S B} / M_{c}\right)^{2}$. Since this is the chirally suppressed term, as argued above, the lagrangian should contain a leading order term, not suppressed by $\mathrm{SU}(3)$ breaking, of order $\left(\Lambda_{C S B} / M_{c}\right)^{2}$. In dimensional regularization, this term is not manifest in the loop calculation, because it arises from matching the full to the effective theory. However, the existence of a lagrangian which is $\mathrm{SU}(3)$ symmetric at leading order and in which current quark mass contributions are suppressed by $m_{s} / \Lambda_{C S B}$ relative to the leading order terms means that the term proportional only to $\left(\Lambda_{C S B} / M_{c}\right)^{2}$ must be there for consistency. This gives us the same conclusion as the previous argument. The only way to prevent large renormalizatons of the tree level operators is to assume that the relevant mass suppression factor is $\Lambda_{C S B} / M$.

Although we have illustrated our point with specific operators, the general argument should be clear. Because we have the same parameters appearing in the chiral lagrangian with and without the heavy mesons, it is inconsistent to choose the scales differently in the case that the heavy meson is and is not present. At the diagrammatic level, the same momentum runs through the heavy meson line and the pseudogoldstone boson lines. The effective theory incorporates the 
scale of the dynamics of the light degrees of freedom, or the momentum with which they can recoil, in the cutoff. The QCD degrees of freedom in the heavy meson are allowed to have momentum up to the cutoff of the effective theory. The largest energy of the virtual degrees of freedom characterizes the expansion. If the derivative expansion of the heavy quark chiral effective theory is indeed applicable up to pion and kaon momenta of the order of the chiral symmetry breaking scale, then consistency demands that this is also the scale which appears in the matrix elements of $1 / M$ suppressed operators, since it is the scale of momenta in the loops. This is true independently of whether the original operator involved derivatives or the gluon field. All the scales should be consistently determined by naive dimensional analysis [4].

It should be emphasized that this is not just a statement about the heavy quark chiral effective theory, although it assumes the existence of this theory, and that it is valid up to the scale $\Lambda_{C S B}$. With this assumption, once we have shown that the scale of the heavy meson lagrangian is $\Lambda_{C S B}$, then the expansion in inverse heavy quark mass requires that this is also the scale which characterizes matrix elements of operators in the heavy quark theory between the physical meson states. If the heavy quark expansion is valid, matrix elements of quark operators suppressed by any given power of $1 / M$ must match onto heavy meson operators suppressed by the same power of $1 / M$. Then, for the matrix elements to agree, which is the requirement of the heavy meson lagrangian, both theories must have the $M$ suppression compensated by the same scale, which we have just demonstrated is $\Lambda_{C S B}$ in the heavy meson lagrangian. This is therefore the scale which characterizes matrix elements in the heavy quark theory as well. For example, the matrix elements of the heavy quark operator $\mathcal{O}^{h}$ between meson states should be approximately equal to that of $\mathcal{O}_{2}{ }^{H}$, implying the relevant scale in the heavy quark theory is also $\Lambda_{C S B}$.

It is of course possible that chiral expansion about the heavy meson does not exist, or that the heavy meson chiral effective theory is not 
valid up to $\Lambda_{C S B}$. There might be a smaller cutoff beyond which the derivative expansion breaks down. It is then this lower cutoff which would set the scale for the $M$ suppressed matrix elements, so that they are small. However, if this scale is indeed significantly lower than $\Lambda_{C S B}$, the heavy meson chiral effective theory would not be of much use, as the kaon mass would be comparable to the cutoff, and even pion loops might not be reliable.

It has been argued [1] that the relevant scale for the cutoff is always some physical degree of freedom, which for the standard chiral lagrangian might be the $\rho$ mass. In the case of the heavy meson lagrangian, this mass might be that of an excited state which has not been included. This state could occur as a virtual intermediate state; the mass difference between this state and the low lying heavy meson states might be what sets the scale of the cutoff, rather than $4 \pi f_{\pi}$. If this is true, it would indicate that the cutoff of the heavy meson chiral lagrangian could be considerably lower than $\Lambda_{C S B}$. For example, the as-yet unobserved states with the total angular momentum of the light degrees of freedom $j=1 / 2$ and with orbital angular momentum $l=1$ have been predicted to be split from the low lying meson states by only $500 \mathrm{MeV}$ [8].

We conclude that it is not possible to consistently treat the matrix elements of heavy quark currents as an expansion in $\Lambda_{Q C D} / M$ and the heavy meson lagrangian as a theory whose cutoff is $\Lambda_{C S B}$. If indeed the chiral lagrangian is valid up to the chiral symmetry breaking scale, this also sets the scale for the mass suppressed matrix elements. Alternatively, the cutoff for the validity of the heavy quark chiral effective theory could be a much lower scale. This would however cast doubt on the utility of the heavy quark chiral lagrangian. 


\section{Discussion and Conclusions}

The implications of this result are unclear. It was already known that there are potential problems with treating a $c$ quark as heavy. If there are various small factors which go the right way, higher order terms in $m_{c}$ might be as small as desired by practitioners of the heavy quark theory. However, it is clear that at least formally, consistency of the heavy quark and chiral expansions requires that heavy quark operators be suppressed by $\Lambda_{C S B} / M$.

It is interesting to note that sum rule calculations have indeed yielded some large values for $\Lambda$ for particular operators. For example, the matrix element of $\mathcal{O}_{1}$ between heavy mesons was defined in ref. [11] as $2 M \lambda_{1}$, where QCD sum rules [11] gave $\lambda_{1} \approx 1 \mathrm{GeV}^{2}$. Other estimates have been done [10] based on a constituent quark model. Since the mass of the constituent quark lies squarely between the QCD and chiral symmetry breaking scale, it can be said to fit with either assumption for $\Lambda$. The spin splitting of the mesons indicates a QCD scale which again lies in between $\Lambda_{Q C D}$ and $\Lambda_{C S B}$.

Probably the most important reason for a better determination of $\Lambda$ is to determine how accurately we can hope to extract KM angles using the heavy quark effective theory. Falk and Neubert [10] did a detailed analysis based on a constituent quark model and QCD sum rule estimates of the various matrix elements, where they find 1 to $3 \%$ corrections from mass suppressed operators. Our results would indicate that comparable errors are incurred just by neglecting the $\mathrm{SU}(3)$ violating contributions to the matrix elements proportional to the strange quark mass. If these are indeed suppressed corrections to leading order operators, the error in the extraction of $V_{c b}$ could be substantially larger than a few percent.

If it is indeed true that the matrix element of the mass suppressed operator is set by the chiral symmetry breaking scale, it would appear that the heavy quark expansion is useless for an extraction of $V_{c b}$. However, if we are lucky, this might not be the case. In the particular 
model for the higher order coefficients employed by Falk and Neubert, there were fortuitous cancellations which made the effects of higher order terms small. However, their work also indicates some universal factors which make it conceivable that one could hope to extract the KM angle at the $10-20 \%$ level. First is the fact that the heavy quark expansion is really an expansion in $1 / 2 M$. Of course this factor of 2 can be compensated by 2's in chiral coefficients, but if we are lucky, the coefficients might all be less than unity (after dimensional analysis factors of order of $1 \mathrm{GeV}$ have been extracted). In this case, $1 / M^{2}$ corrections might be suppressed by $\left(1 \mathrm{GeV} / 2 M_{c}\right)^{2} \approx 10 \%$. Even for $B \rightarrow D$, which is not protected by Luke's theorem [13] so that $1 / M$ corrections are present, there is the Voloshin-Shifman factor [12], $S=\left(m_{B}-m_{D}\right)^{2} /\left(m_{B}+m_{D}\right)^{2} \approx 0.23$, multiplying the leading $1 / M$ corrections. Therefore, these corrections might also be at the level of $10 \%$.

The considerations of this paper also apply to the baryon lagrangian. This would severely compromise the utility of the baryon lagrangian. Here, the expansion parameter would be expected to be $\Lambda_{C S B} / 2 M_{\text {nucleon }}$, which is not small.

It might well be that the mass suppressed matrix elements are smaller than suggested in this note. Alternatively, the chiral lagrangian for heavy mesons might not be valid, or fail at a scale considerably lower than the standard chiral symmetry breaking scale. However, it is clear that until there is a firm argument that the estimate based on chiral loop estimates is incorrect, the uncertainty in extracting KM angles, even if it occurs only at $1 / M^{2}$, is very large. It would be very useful to have measurements of mass suppressed heavy quark matrix elements to determine whether mass suppressed operators can be as large as implied by these estimates. 


\section{Acknowledgements}

We thank Adam Falk for useful discussions. This work is supported in part by funds provided by the U. S. Department of Energy (D.O.E.) under contract \#DE-AC02-76ERO3069.

\section{References}

[1] See H. Georgi (1991) Lectures presented at the Theoretical Advanced Study Institute, Boulder (World Scientific), to be published; M. Wise (1991)Lectures presented at the Lake Louise Winter Institute, Caltech preprint CALT-68-1721, B. Grinstein (1991) Proc. High Energy Phenomenology Workshop, Mexico City, eds. R. Huerta and M. Perez, SSCL preprint 91-17, and refs. therein.

[2] N. Isgur and M. Wise, Phys. Lett. B 237 (1990) 527; A. Falk, B. Grinstein, and M. Luke, Nucl. Phys. B 357 (1991) 185.

[3] M. Wise, Phys. Rev. D45 (1992) 3021.

[4] A. Manohar and H. Georgi, Nucl. Phys. B 234 (1984) 189; H. Georgi and L. Randall, Nucl. Phys. B 276 (1986) 241.

[5] H. Georgi, "Weak Interactions and Modern Particle Theory", Benjamin/Cummings Publishing Co., Inc. (1984).

[6] H. Georgi HUTP-92/A049

[7] R. S. Chivukula, M. Dugan, and M. Golden , BUHEP-92-18.

[8] S. Godfrey and N. Isgur, Phys. Rev. D32 (1985) 189.

[9] L. Randall and E. Sather, MIT-CTP-2166

[10] A. Falk and M. Neubert, SLAC-PUB-5897, 1992.

[11] M. Neubert, Phys. Rev. D45, 2451 (1992).

[12] M. Voloshin and M. Shifman, Yad. Fiz. 45, 463 (1987) [Sov. J. Nucl. Phys. 45, 292 (1987); 47, 801 (1988) [47, 511 (1988)].

[13] M. Luke, Phys. Lett. B 349, 598 (1991). 